\title{
Biomimicking lubrication superior to fish skin using responsive hydrogels
}

\author{
Yang $\mathrm{Wu}^{1,2}$, Xiaowei Pei ${ }^{1}$, Xiaolong Wang ${ }^{1}$, Yongmin Liang ${ }^{1}$, Weimin Liu ${ }^{1}$ and Feng Zhou ${ }^{1}$ \\ The slippery mucus produced by fish skin is important to protect fish against predator attack, allowing fish to swim faster and \\ remain elusive because of the ultra-low coefficient of friction (COF) of fish skin. To mimic this slick skin, responsive hydrogels \\ that respond to external stimuli, including $\mathrm{pH}$ and temperature, were prepared. These hydrogels were found to perform better \\ than fish skin: not only was an ultra-low COF achieved but multiple tunable COFs from ultra-low to ultra-high were discovered \\ using sequential regulation of $\mathrm{pH}$ and temperature. The tunable COF was achieved through conformational changes in the \\ molecular chains in the responsive hydrogel that were induced by the external stimuli. Swelling of both $\mathrm{pH}$ - and thermal- \\ responsive polymer chains of the hydrogel resulted in an ultra-low COF; the pH-responsive component, shrink as a result of \\ dehydration caused by a pH change, led to a moderate COF, whereas the two components simultaneous shrink brought out a \\ very high COF. The three levels of COF under different states can be reversibly switched multiple times by sequential regulation \\ of $\mathrm{pH}$ and temperature. This reversible tunability in friction performance is likely to have a significant impact on the design of \\ hydrogel-based actuation devices.
}

NPG Asia Materials (2014) 6, e136; doi:10.1038/am.2014.82; published online 3 October 2014

\section{INTRODUCTION}

Learning from nature is an effective way to obtain new knowledge and prepare new materials. In recent years, numerous successes have been reported, including a superhydrophobic bionic device derived based on the lotus leaf, ${ }^{1}$ a slippery liquid-infused porous surface bionic device derived based on nepenthes ${ }^{2}$ and micro/nanopillar bionic devices with high adhesive force derived based on geckos. ${ }^{3}$ Meanwhile, researchers are also examining biomimicry to improve on superior to nature, ${ }^{4}$ such as superoleophobicity, ${ }^{5,6}$ switchable wetting, ${ }^{7,8}$ and selfhealing, ${ }^{9,10}$ which are only loosely based on the examples from nature.

It is well known that mucus is secreted on fish skin to help fish adapt to the external environment. ${ }^{11}$ Fish skin can be so slippery that it makes fish difficult to catch by hand. Fish mucus (that is, a hydrophilic biopolymer) acts as an excellent lubricant when a person holds a fish. This lubricant has an ultra-low friction coefficient $\left(5 \times 10^{-3}\right.$ or smaller $)$. To mimic the slipperiness of fish skin, hydrogels may be used because of their hydrophilic characteristics. These compounds trap a large amount of water on their gel surfaces, acting as a lubricating film similar to the mucus on fish skin. Artificial hydrogels have achieved even lower coefficients of friction (that is, $10^{-4}$ ) than natural fish skin, as reported by Gong et al. ${ }^{12-14}$ To this extent, biomimicking hydrogels that improve on nature's designs have been realized. However, despite the similar physicochemical properties of these compounds to biological tissues and their potential for biomedical applications, ${ }^{15-17}$ artificial hydrogels are quite distinct from the natural phenomena that they replicate, especially in their controllable attributes. Most organisms have systems that are dedicated to respond to external stimuli; in advanced animals, this system is the nervous system. Under an external stimulus, a neural signal is produced to control a muscle by either contracting it or relaxing it; this type of body control is typically used to escape from dangerous situations or change the animal's condition to adapt to its external surroundings. To improve the controllability of a system, numerous stimulus-sensitive hydrogels have been synthesized by hybridizing the responsive molecules/monomers in the gel system. ${ }^{18,19}$ Because of the hydration/dehydration interaction between the hydrogel and water molecules, the shape or surface physicochemical performance changes in response to the external stimuli; the sensitive hydrogels have been observed to remain in a swollen or collapsed state under diverse conditions, through which controllable capacity was realized. However, apart from the utilization of these typical characteristics of stimulus-sensitive hydrogels for drug release $\mathrm{e}^{20,21}$ and chemical sensors or actuators, ${ }^{8,22}$ tribological applications of stimulus-sensitive hydrogels have been rarely targeted even though they are the artificial materials most similar to fish skin.

Recently, Chang et al. ${ }^{23}$ investigated the switchable tribological properties of a poly $\mathrm{N}$-isopropyl-acrylamide (pNIPAM) gel induced by alternating exposure to water/methanol solvents at room temperature (rt). Very recently, the authors of this paper realized a switchable coefficient of friction $(\mathrm{COF})$, from ultra-low $(\sim 0.02)$ to ultra-high

${ }^{1}$ State Key Laboratory of Solid Lubrication, Lanzhou Institute of Chemical Physics, Chinese Academy of Sciences, Lanzhou, P. R. China and ${ }^{2}$ University of Chinese Academy of Sciences, Beijing, P. R. China

Correspondence: Professor X Wang or Professor F Zhou, State Key Laboratory of Solid Lubrication, Lanzhou Institute of Chemical Physics, Chinese Academy of Sciences, 18 Tianshui Middle Road, Lanzhou, Gansu 730000, P. R. China.

E-mail: wangx|@licp.cas.cn or zhouf@licp.cas.cn

Received 26 March 2014; revised 8 May 2014; accepted 9 July 2014 
( $>0.1$ ) values, by using a graphene-pNIPAM composite hydrogel, ${ }^{24}$ where the graphene oxide (GO) and pNIPAM strengthen and sensitize the compound, respectively. Transformation of the intermolecular and intramolecular H-bonding due to temperature alternation across the lower critical solution temperature (LCST) ${ }^{25}$ resulted in the switching of the COF. However, the fact that friction of this type of hydrogel can be switched only between two points keeps its applications limited. Hydrogels with multiple tunability of their COF between ultra-low and ultra-high level are therefore much more desirable for practical applications; however, challenges remain. Conformational changes of polyionic grafts caused by external stimuli such as $\mathrm{pH}$ and temperature can result in two levels of switching of nano-friction. ${ }^{26}$ Accordingly, the challenge of incorporating other sensitive monomers into the previous graphene-pNIPAM gel system is examined to realize the multiple states of friction; this is biomimicry beyond fish skin. As a proof-of-concept, a $\mathrm{pH}$-sensitive monomer, sodium methacrylate (NaMA) and 2-(dimethylamino)ethyl methacrylate (DMAEMA) were introduced, which endow the thermal-sensitive hydrogel with a $\mathrm{pH}$-sensitive character, resulting in hydrogels with dual responsiveness. Altering the $\mathrm{pH}$ and temperature of the environment sequentially resulted in hydrogels with varying and regulatable COFs from ultra-low to high values.

\section{EXPERIMENTAL PROCEDURE}

\section{Chemicals and materials}

$N$-isopropylacrylamide(NIPAM), NaMA, and $N, N, N^{\prime}, N, N^{\prime \prime}$-pentamethyldiethylene-triamine were purchased from Alfa Aesar (Shanghai, China) DMAEMA was purchased from J\&K Chemicals (Beijing, China). $\mathrm{N}, \mathrm{N}$ Methylene-bisacrylamide (BIS) and ammonium persulfate were purchased from Shanghai Chemical Reagent Company (Shanghai, China). All chemicals are analytical reagents and were used as received.

\section{Synthesis of composite hydrogels with dual responsiveness}

GO sheets were prepared according to the method previously reported. ${ }^{26,27}$ The concentration of GO solution was prepared at a concentration of $1.5 \mathrm{mg} \mathrm{ml}^{-1}$. Composite hydrogels were prepared using initial solutions consisting of monomer (NIPAM, NaMA or DMAEMA), crosslinker (BIS), initiator (ammonium persulfate) and GO. A typical process was as follows: $0.66 \mathrm{~g}$ of NIPAM, $0.132 \mathrm{~g}$ of NaMA, $40 \mathrm{mg}$ of BIS, $30 \mathrm{mg}$ of ammonium persulfate and $30 \mu \mathrm{l}$ of $\mathrm{N}$, $N, N^{\prime}, N, N^{\prime \prime}$-pentamethyldiethylene-triamine were added to $12 \mathrm{ml}$ of GO solution and stirred at $0^{\circ} \mathrm{C}$ for $15 \mathrm{~min}$ to form a stable dispersion. Next, the dispersion was poured into a cubic mold and sealed. The polymerization was conducted in the mold for $10 \mathrm{~h}$ at rt. The prepared hydrogel was then dipped into a phosphate-buffered solution until swelling equilibrium was reached. To investigate the dual responsiveness of the compound, hydrogels with different NIPAM and NaMA content were prepared, and these are denoted pNIPAM $-\mathrm{NaMA}_{y}$, where the subscripts $x$ and $y$ represent the mass ratio.

\section{Frictional test experiment}

The frictional test device used for this investigation was a 14-FW statnamic tribometer (HEIDON, Tokyo, Japan). Following the previous report, ${ }^{24}$ the COF was obtained with the reciprocating mode of this device and the COF curve was obtained with the single-track mode of this device. The frictional test was conducted in a phosphate-buffered solution in face-to-face contact mode, and the upper friction probe was covered in the same hydrogel surface. The testing temperature was controlled by a computer, and the stress and sliding speed were set to $1 \mathrm{~N}$ and $200 \mathrm{~mm} \mathrm{~min}^{-1}$, respectively.

\section{RESULTS AND DISCUSSION}

Experimentation began with a friction test of a piece of fresh catfish skin that was $20 \mathrm{~cm}$ long from the body of the fish, as shown in Figure 1a. It was found that the large amount of mucus on catfish skin makes it very slippery, with an average COF of $5 \times 10^{-3}-7 \times 10^{-3}$ not only in distilled water but also in the buffer solution at high temperatures $\left(40^{\circ} \mathrm{C}\right)$. A series of $\mathrm{pH}$-thermal-sensitive hydrogels were then prepared for testing as a proof-of-concept. As shown in Figure $1 \mathrm{~b}$, the $\mathrm{pH}$-sensitive hydrophilic monomer NaMA was introduced into the thermosensitive pNIPAM gels. Owing to the $\mathrm{pH}$-sensitive component pNaMA in the thermal hydrogel, the composite hydrogel showed both $\mathrm{pH}$ - and thermal-responsive characteristics. The pKa of NaMA is 4.8 , indicating that the pNaMA chains would collapse at an acidic $(\mathrm{pH}<4.8)$ and swell in weakly acidic or alkaline $\mathrm{pH}$ surroundings $(\mathrm{pH}>4.8)$. Figure $1 \mathrm{~b}$ synoptically describes the continuous change in the gel state of the $\mathrm{pH}$-thermal hybrid hydrogel with the two stimuli, $\mathrm{pH}$ and temperature. $\mathrm{pH}$ alteration causes the pH-sensitive pNaMA segments to swell or collapse, which influences the physicochemical properties of the hydrogel. On this basis, once the surrounding temperature crosses the LCST of pNIPAM, a new stage appears, with different characteristics of the gel. The conformation change in the gel at each stage allows tunable friction performance.

The prediction above was verified by friction tests: the regulation of the $\mathrm{COF}$ of the $\mathrm{pH}$-thermal gel from ultra-low to moderate level, and then to ultra-high values were achieved by changing the $\mathrm{pH}$ of the solution and the temperature successively. Figure 1c shows the COF curves of the pNIPAM ${ }_{11}-\mathrm{NaMA}_{3}$ gel under different $\mathrm{pH}$ environments and temperatures. In a neutral solution and below the LCST of NIPAM, the two polymer segments of the gel both showed a swollen state. Abundant water was stored in the gel network, which resulted in a stable water film between the gel friction pair, acting as a lubrication layer with an ultra-low and stable COF (0.05, black line in Figure 1c). When the $\mathrm{pH}$ of the surroundings became acidic $(\mathrm{pH}=2)$, the pNaMA segment of the gel collapsed. Some of the trapped water was expelled, resulting in a reduced water film between the gel surfaces with a COF rising to a moderate level near 0.16 (red line in Figure 1c). When the temperature was then increased to $32^{\circ} \mathrm{C}$ in acidic surroundings, the COF abruptly increased to 1.0 and became unstable (green line in Figure 1c). This was attributed to both the collapsed polymer segments in the gel under this condition. Because most of the water was squeezed out, the water film between the friction pair was damaged, and the surfaces of the friction pair thoroughly contacted with almost no lubricants, leading to a sharp increase in the COF and the resulting unstable curves. Notably, the $\mathrm{pH}$ and temperature responses of the pNIPAM-NaMA gels are fully reversible. Figure $1 \mathrm{~d}$ shows the reversible friction changes in the pNIPAM $_{11}-\mathrm{MAA}_{3}$ gel. When the $\mathrm{pNIPAM}_{11}-\mathrm{MAA}_{3}$ gel frictional pairs were examined in the friction test at $\mathrm{pH}=7$ and $\mathrm{pH}=2, \mathrm{rt}$; and $\mathrm{pH}=2$, at $32^{\circ} \mathrm{C}$ successively, its $\mathrm{COF}$ was increased from an ultra-low value $(\sim 0.05)$ to a moderate $(\sim 0.16)$ and finally to a very high level $(>1.0)$. This change in COF has been described for a dual-responsive hydrogel (Figure 1d).

After changing the concentration of NaMA in the gel system, the resultant hydrogels showed different COF response behaviors to the surrounding $\mathrm{pH}$ and temperature. Two other typical gels, pNIPAM $_{5}-\mathrm{NaMA}_{1}$ and $\mathrm{pNIPAM}_{5}-\mathrm{NaMA}_{2}$, with lower and higher NaMA fractions than PNIPAM $_{11}-\mathrm{MAA}_{3}$ gel were also investigated, respectively (Figures $2 \mathrm{a}$ and $\mathrm{b}$ ). For the low NaMA content gel $\left(\mathrm{pNIPAM}_{5}-\mathrm{NaMA}_{1}\right)$, the COF increased from 0.04 to 0.07 when altering the $\mathrm{pH}$ from 7 to 2 , and then to 0.12 when increasing the testing temperature to $32^{\circ} \mathrm{C}$ (Figure 2a). However, for the high NaMA content gel $\left(\mathrm{pNIPAM}_{5}-\mathrm{NaMA}_{2}\right)$, its COF increased from 0.05 to 0.2 and then to 1.2 when changing the $\mathrm{pH}$ and temperature in the same sequence, respectively (Figure $2 \mathrm{~b}$ ). It was also found that the hybrid hydrogels with different NaMA fractions had different responses to temperature in neutral and acidic surroundings. The COF of other 


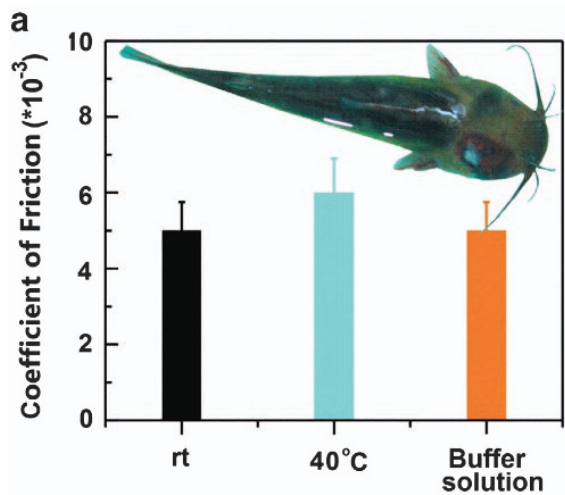

b
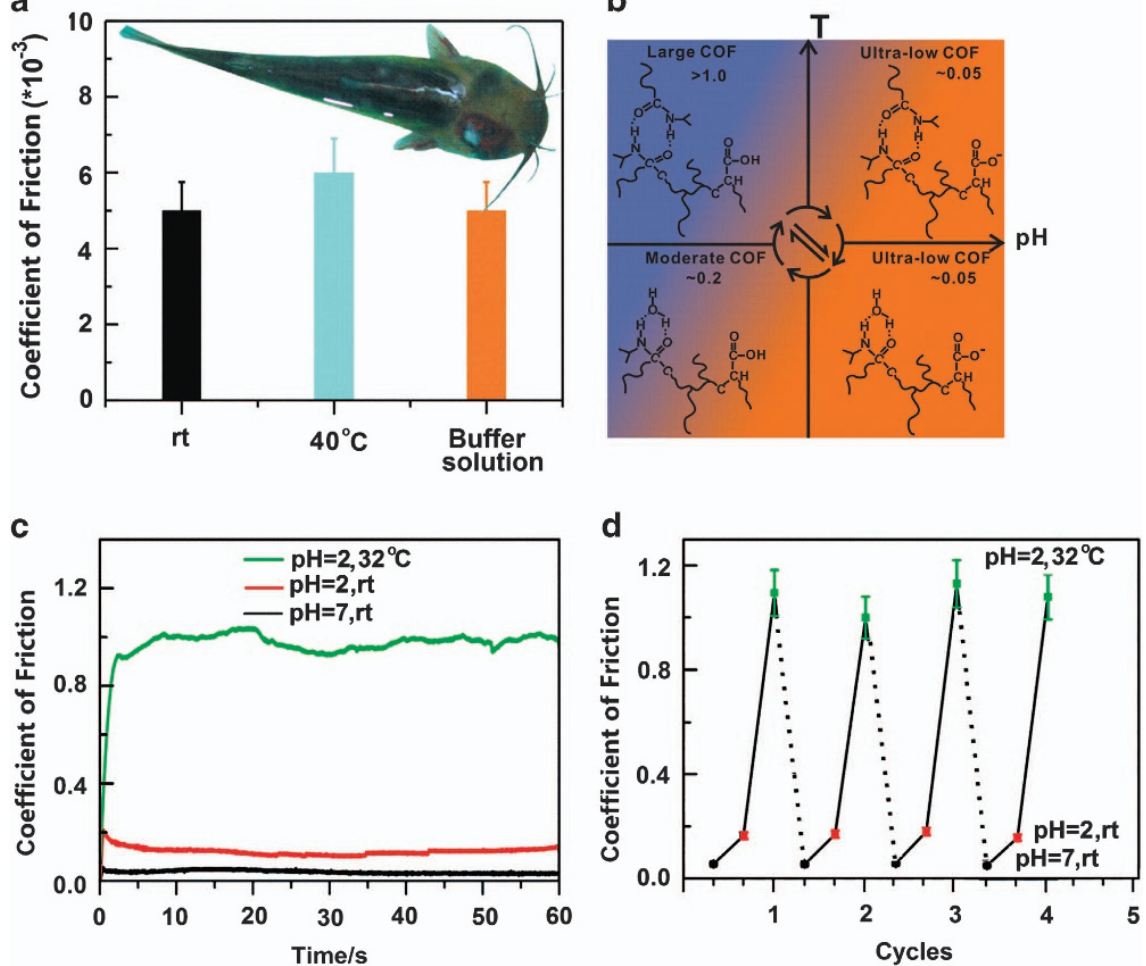

Figure 1 (a) Digital image of a catfish and the corresponding COF values of the catfish skin under different conditions; (b) Schematic illustration of the continuous regulation of the $\mathrm{COF}$ of the $\mathrm{pH}$-thermal-sensitive composite hydrogels owing to the reversible swelling and collapse cycles induced by the two stimuli, $\mathrm{pH}$ and temperature; (c) The COF curves of the pNIPAM $11-\mathrm{NaMA}_{3}$ gel at $\mathrm{pH}=7$ and 2, rt, and $\mathrm{pH}=2$ at $32{ }^{\circ} \mathrm{C}$; and (d) The switchable $\mathrm{COF}$ of the pNIPAM $_{11}-\mathrm{NaMA}_{3}$ gel with the stimuli of $\mathrm{pH}$ and temperature. COF, coefficient of friction; NaMA, sodium methacrylate; pNIPAM, poly N-isopropylacrylamide; rt, room temperature.
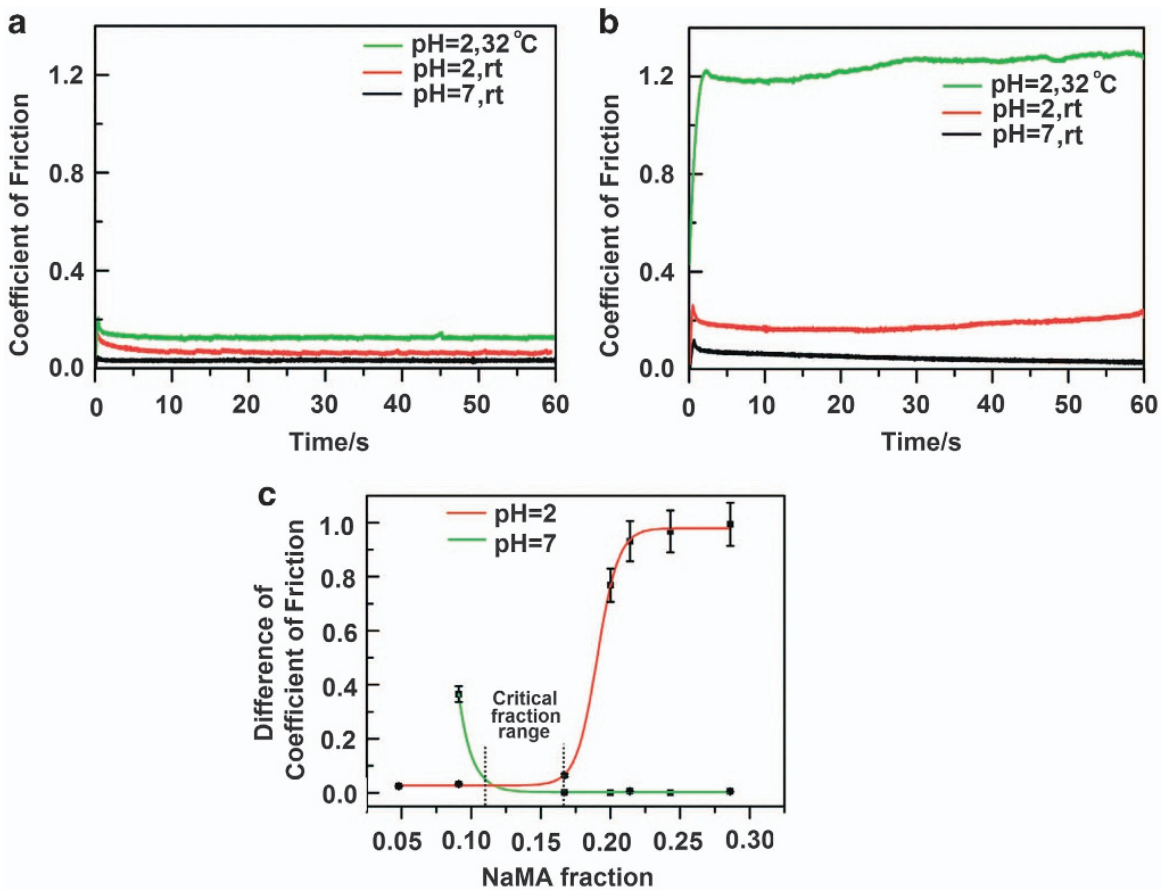

Figure 2 The COF curves of (a) the pNIPAM $5-\mathrm{NaMA}_{1}$ gel; (b) the pNIPAM $-\mathrm{NaMA}_{2}$ gel at $\mathrm{pH}=7$, rt; $\mathrm{pH}=2$, rt; and $\mathrm{pH}=2$ at $32^{\circ} \mathrm{C}$; and (c) The $\Delta \mathrm{COF}$ of the pNIPAM-NaMA gels in neutral and acidic surroundings. COF, coefficient of friction; NaMA, sodium methacrylate; pNIPAM, poly $N$-isopropyl-acrylamide; rt, room temperature. 
hydrogels with different NaMA mass fractions at room and elevated temperatures in neutral and acidic surroundings are shown in Supplementary Figure S1. The difference between the COF at rt and that at an elevated temperature $(\Delta \mathrm{COF})$ exhibits a nearly opposite trend in neutral and acidic surroundings (Figure 2c). In neutral surroundings, the $\triangle \mathrm{COF}$ changed from 0.4 to near zero when the mass fraction of NaMA was increased from $9.1-16.7 \%$ or higher. Although in acidic surroundings, it increased from zero to near 1.0 with an increasing mass fraction of NaMA, the turning point was at a NaMA fraction of $16.7 \%$. Moreover, regardless of the $\mathrm{pH}$ of the surroundings, there were no abrupt changes in the $\triangle \mathrm{COF}$ for NaMA fractions between 12.0 and $16.5 \mathrm{wt} \%$ (Figure 2c). The result can guide to prepare the desired responsive hydrogels, namely, if one wants to prepare the hydrogels with dual responsiveness; the NaMA fraction of the hydrogels must not be in the critical fraction range (that is, $12.0-16.5 \%)$ and vice versa.

Our previous report found similar phenomena: ${ }^{24}$ a slight amount of non-thermal monomer introduced into thermal pNIPAM gels caused large variations in the COF of hydrogels above the LCST. To better understand, three hydrogels with NaMA mass fractions, pNIPAM $_{10}-\mathrm{NaMA}_{0.5}, \mathrm{pNIPAM}_{10}-\mathrm{NaMA}_{1}$ and $\mathrm{pNIPAM}_{10}-\mathrm{NaMA}_{2}$, were subjected to further friction investigation in acidic and neutral solutions at rt, 34 and $80^{\circ} \mathrm{C}$ (Figures $3 \mathrm{a}$ and b), respectively. In acidic surroundings, the COFs of all three samples increased drastically when the temperature was increased from 31 to $34^{\circ} \mathrm{C}$, whereas this had almost no effect in neutral surroundings. For example, the COF of the pNIPAM $_{10}-\mathrm{NaMA}_{1}$ gel increased from 0.05 to 0.40 between 31 and $34^{\circ} \mathrm{C}$ in a $\mathrm{pH}=2$ solution, yet almost no changes were observed in this temperature range in a $\mathrm{pH}=7$ solution. Accordingly, in neutral surroundings, the COF of this gel was observed to change from 0.05 to 0.42 between 44 and $54^{\circ} \mathrm{C}$. We attributed the difference to the different hydration ability of pNaMA in acidic and neutral solutions. As shown in Figure 1b, the pNaMA was in collapse in acid, leading to limited hydration. Therefore, when the pNIPAM chains shrank at an elevated temperature, its negative effects dominated, resulting in a drastic increase in COF. However, in neutral surroundings, pNaMA had excellent hydration, which counteracted the negative effects caused by the shrinking of PNIPAM when the temperature increased and led to a delayed but commensurate increase of the COF. As shown in Figure $3 \mathrm{~b}$, the sharp increase in COF occurred at 34 and $45^{\circ} \mathrm{C}$ for pNIPAM $_{10}-\mathrm{AM}_{0.5}$ and $\mathrm{pNIPAM}_{10}-\mathrm{AM}_{1}$, respectively, and occurred over 6 and $10^{\circ} \mathrm{C}$ from 0.05 to 0.42 , respectively. When the NaMA was increased to $16.7 \%$ or more in the $\mathrm{pNIPAM}_{10}-\mathrm{NaMA}_{2}$ gel, the COF became independent of the temperature of the neutral solution. Even if the temperature was increased to $80^{\circ} \mathrm{C}$, the $\mathrm{COF}$ of this gel remained at a low and constant value near 0.04 . This indicates that the excellent hydration of NaMA, when its fraction is $16.7 \mathrm{wt} \%$ or more, is completely dominant at all tested temperatures in a neutral solution. These results verify that responsive hydrogels with controllable responses to $\mathrm{pH}$ and temperature can be readily realized by simply changing the $\mathrm{pH}$-sensitive monomer fraction in the $\mathrm{pH}$-thermal hydrogel system.

Theoretically, many dual- and even multi-responsive hydrogels can be prepared using this concept. The other proof-of-concept sample incorporated DMAEMA into the pNIPAM gel system. In contrast to the pNaMA in the pNIPAM-NaMA gels, pDMAEMA chains swell in acidic surroundings because of the quaternization of the tertiary amine group, and they shrink in alkaline solutions. The structure of the pNIPAM-DMAEMA hybrid hydrogel, its $\mathrm{pH}$-thermal-response behaviors and its friction responses, are shown in Supplementary Figure S2. Similar to the pNIPAM-NaMA gels, the pNIPAM-DMAEMA gels also switched from an ultra-low to ultra-high value by means of changing the $\mathrm{pH}$ of the solution and the testing temperature. Figure $4 \mathrm{a}$ shows the COF curves of the $\mathrm{pNIPAM}_{28}-\mathrm{DMAEMA}_{1}$ gel when the surrounding $\mathrm{pH}$ and temperature were changed. In an acidic solution, the pDMAEMA chains swelled and absorbed water molecules, leading to a low stable COF near 0.05 (black line in Figure 4a) at rt. When the surroundings were changed to an alkaline solution, the COF curve rose to near 0.25 (red line in Figure 4a). And finally, when the testing temperature was increased to only $30{ }^{\circ} \mathrm{C}$, which is still below the LCST of NIAPM, the COF of pNIAPM $_{28}-$ DMAEMA $_{1}$ gel unexpectedly reached 0.67 (green line in Figure 4a). For this situation, the salt effect on the thermal-sensitive gel should be recalled because the phosphate buffer solution was used in this experiment. ${ }^{28,29}$ In salt solutions, the pNIPAM has a lower cloud point temperature, ${ }^{30,31}$ which means that the LCST of the pNIPAM dropped. When the salt concentration reaches a certain level, the LCST can drop below rt or even below $5{ }^{\circ} \mathrm{C} .{ }^{28}$ Therefore, in an alkaline solution even at $30^{\circ} \mathrm{C}$, parts of collapsed pNIPAM chains still led to a high COF. Similar to the pNIPAM-NaMA gel system, the DMAEMA content had effects on the response behavior of the resultant pNIPAM-DMAEMA gels. Investigation of the other two pNIAPM-DMAEMA gels with higher DMAEMA fractions than the $\mathrm{pNIAPM}_{28}-\mathrm{DMAEMA}_{1}$ gel verified that the increase of the DMAEMA fraction can weaken the salt effect of the pNIPAM gel (Figures $4 \mathrm{~b}$ and c). Similarly, a reversible switchable COF of pNIAPM-DMAEMA gels also can be obtained by changing the solution $\mathrm{pH}$ and temperature. For example, the COF of the pNIAPM $_{28}-$ DMAEMA $_{1}$ gel was able to be switched from 0.05 to 0.25 and then to 0.7 many times without any damage to the gel (Figure 4d). Friction tests of several pNIPAM-DMEMA gels clearly
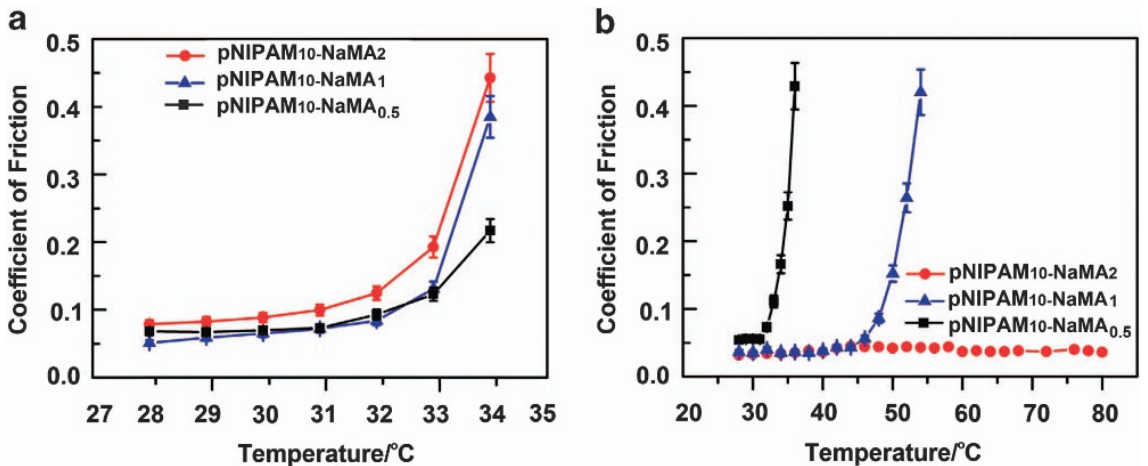

Figure 3 The $\mathrm{COF}$ of three hydrogels as a function of the testing temperature in (a) $\mathrm{pH}=2$ and (b) $\mathrm{pH}=7$ surroundings. 


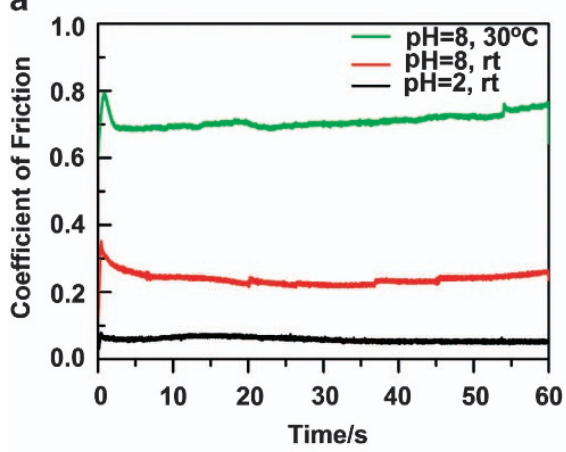

C



b

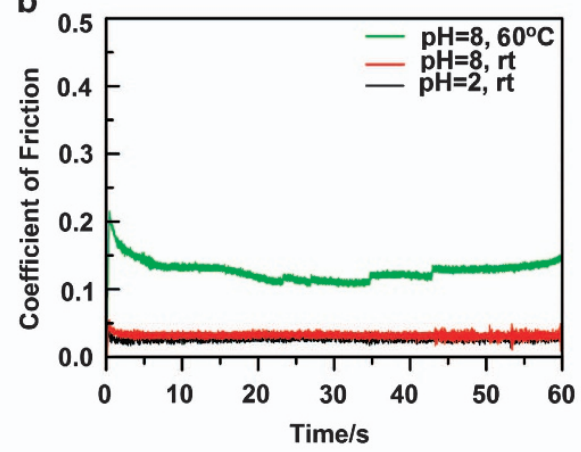

d

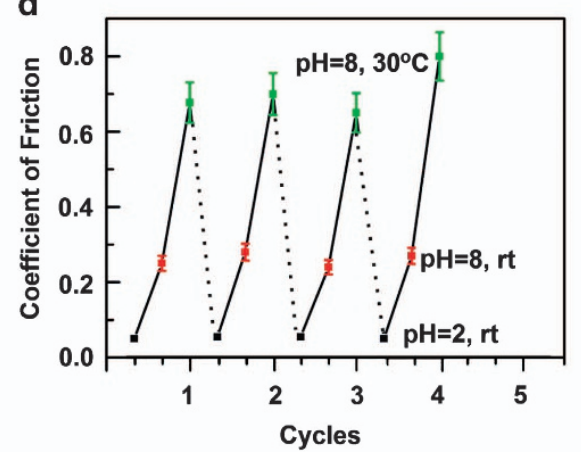

Figure 4 The COF curves of (a) the pNIPAM $28-D_{2 M A E M A}$ gel; (b) the pNIPAM $10-$ DMAEMA $_{1}$ gel; (c) the pNIPAM $13-$ DMAEMA $_{3}$ gel under three conditions; and (d) the switchable COF cycles of pNIPAM $28-$ DMAEMA $_{1}$ gel. COF, coefficient of friction; DMAEMA, 2-(dimethylamino)ethyl methacrylate; pNIPAM, poly $\mathrm{N}$-isopropyl-acrylamide.

showed that the response of the gels to temperature in acidic and alkaline solutions were highly dependent on the DMAEMA fraction in the system (Supplementary Figure S3). Because the pDAMEMA chains were swollen in acidic solutions, even $2.0 \mathrm{wt} \%$ of DAMEMA in the hybrid hydrogel had a significant influence on the COF by postponing the transformation temperature and slowing down the transformation process (Supplementary Figure S3a); however, the pNIPAM $_{10}-$ DMEMA $_{1}$ gel with $9.1 \mathrm{wt} \%$ DMAEMA kept a constant COF near 0.03 even at $80^{\circ} \mathrm{C}$. Conversely, in alkaline surroundings, the transformation temperature of the COF increased with the DMAEMA fraction in the hybrid hydrogel (Supplementary Figure S3b).

\section{CONCLUSION}

In conclusion, a general feasible approach for achieving hybrid hydrogels with dual- and even multi-responsive characteristics in response to stimuli has been developed. Two series of $\mathrm{pH}$-thermalsensitive hydrogels were demonstrated by incorporating NaMA or DMAEMA into a thermally responsive pNIPAM gel. These hybrid hydrogels had an ultra-low COF (that is, near 0.05). Although the lowest COF found was an order of magnitude higher than the slippery mucus on natural fish skin. The stepwise switching of the COF between 0.05 and 1.2 by sequentially regulating the $\mathrm{pH}$ and temperature of the solution makes this improvement on nature's design possible. Importantly, this kind of multi-step regulation of the COF was reversible many times without damaging the gels. Moreover, it was also found that the transformation temperature of the COF of hybrid hydrogels can be regulated by altering the mass fraction of the $\mathrm{pH}$-sensitive monomer. Using this design philosophy, various dual- or multi-responsive hydrogels with reversible COF tunability can be prepared, in principal, and are promising materials for a broad range of applications. The ability to change the COF of these hydrogels based on external stimuli make them particularly useful in applications that cannot provide an external power source. Furthermore, different stimuli can produce widely varying COFs to meet practical requirements, such as controlling velocity or selection of a body. ${ }^{24}$ These gels with multiple or even continuously regulated and reversible COFs will have a significant impact on the design of special coatings and surfaces for devices in micro-mechanics, actuators and sensors in the near future.

\section{CONFLICT OF INTEREST}

The authors declare no conflict of interest.

\section{ACKNOWLEDGEMENTS}

We are grateful for financial support from the NSFC (21125316, 21434009, 51335010), 973 Project (2013CB632300) and the Key Research Program of the Chinese Academy of Sciences (grant no. KJZD-EW-M01).

1 Blossey, R. Self-cleaning surfaces-virtual realities. Nat. Mater. 2, 301-306 (2003).

2 Wong, T. S., Kang, S. H., Tang, S. K., Smythe, E. J., Hatton, B. D., Grinthal, A. \& Aizenberg, J. Bioinspired self-repairing slippery surfaces with pressure-stable omniphobicity. Nature 477, 443-447 (2011).

3 Lee, H., Lee, B. P. \& Messersmith, P. B. A reversible wet/dry adhesive inspired by mussels and geckos. Nature 448, 338-341 (2007).

4 Liu, X., Liang, Y., Zhou, F. \& Liu, W. Extreme wettability and Tunable adhesion biomimicking beyond Nature? Soft Matter 8, 2070-2086 (2012).

5 Wu, W., Wang, X., Wang, D., Chen, M., Zhou, F., Liu, W. \& Xue, Q. Alumina nanowire forests via unconventional anodization and super-repellency plus low adhesion to diverse liquids. Chem. Commun. (Camb) 1043-1045 (2009).

6 Tuteja, A., Choi, W., Ma, M., Mabry, J. M., Mazzella, S. A., Rutledge, G. C., McKinley, G. H. \& Cohen, R. E. Designing superoleophobic surfaces. Science 318, 1618-1622 (2007). 
7 Sun, T., Wang, G., Feng, L., Liu, B., Ma, Y., Jiang, L. \& Zhu, D. Reversible switching between superhydrophilicity and superhydrophobicity. Angew. Chem. Int. Ed. Engl. 43, 357-360 (2004).

8 Xin, B. \& Hao, J. Reversibly switchable wettability. Chem. Soc. Rev. 39, 769-782 (2010).

9 Toohey, K. S., Sottos, N. R., Lewis, J. A., Moore, J. S. \& White, S. R. Self-healing materials with microvascular networks. Nat. Mater. 6, 581-585 (2007).

10 Wang, X., Liu, X., Zhou, F. \& Liu, W. Self-healing superamphiphobicity. Chem. Commun. (Camb) 47, 2324-2326 (2011).

11 Shephard, K. L. Functions for fish mucus. Rev. Fish Biol. Fisher 4, 401-429 (1994) .

12 Gong, J. P. Friction and lubrication of hydrogels? its richness and complexity. Soft Matter 2, 544-552 (2006).

13 Kaneko, D., Tada, T., Kurokawa, T., Gong, J. P. \& Osada, Y. Mechanically strong hydrogels with ultra-low frictional coefficients. Adv. Mater. 17, 535-538 (2005).

14 Gong, J. P., Kurokawa, T., Narita, T., Kagata, G., Osada, Y., Nishimura, G. \& Kinjo, M. Synthesis of hydrogels with extremely low surface friction. J. Am. Chem. Soc. 123, 5582-5583 (2001).

15 Vermonden, T., Censi, R. \& Hennink, W. E. Hydrogels for protein delivery. Chem. Rev. 112, 2853-2888 (2012)

16 Lee, K. Y. \& Mooney, D. J. Hydrogels for tissue engineering. Chem. Rev. 101, 1869-1879 (2001).

17 Grant, C., Twigg, P., Egan, A., Moody, A., Smith, A., Eagland, D., Crowther, N. \& Britland, S. Poly(vinyl alcohol) hydrogel as a biocompatible viscoelastic mimetic for articular cartilage. Biotechnol. Prog. 22, 1400-1406 (2006).

18 Ahn, S. K., Kasi, R. M., Kim, S. C., Sharma, N. \& Zhou, Y. X. Stimuli-responsive polymer gels. Soft Matter 4, 1151-1157 (2008).

19 Tokarev, I. \& Minko, S. Stimuli-responsive hydrogel thin film. Soft Matter 5 511-524 (2009).

20 Qiu, Y. \& Park, K. Environment-sensitive hydrogels for drug delivery. Adv. Drug. Deliver. Rev. 64, 49-60 (2012).

21 Brun-Graeppi, A. K. A. S., Richard, C., Bessodes, M., Scherman, D. \& Merten, O.-W. Thermoresponsive surfaces for cell culture and enzyme-free cell detachment. Prog. Polym. Sci. 35, 1311-1324 (2010)

22 Calvert, P. Hydrogels for soft machines. Adv. Mater. 21, 743-756 (2009) .
23 Chang, D. P., Dolbow, J. E. \& Zauscher, S. Switchable friction of stimulus-responsive hydrogels. Langmuir 23, 250-257 (2007).

24 Wu, Y., Cai, M., Pei, X., Liang, Y. \& Zhou, F. Switching friction with thermalresponsive gels. Macromol. Rapid Comm. 34, 1785-1790 (2013).

25 Liang, L., Feng, X., Liu, J., Rieke, P. C. \& Fryxell, G. E. Reversible surface properties of glass plate and capillary tube grafted by photopolymerization of $\mathrm{N}$-isopropylacrylamide. Macromolecules 31, 7845-7850 (1998).

26 Nordgren, N. \& Rutland, M. Tunable nanolubrication between dual-responsive polyionic grafts. Nano Lett. 9, 2984-2990 (2009).

27 Hummers, W. S. \& Offeman, R. E. Preparation of graphitic oxide. J. Am. Chem. Soc. 80, 1339 (1958).

28 Shen, J., Yan, B., Li, T., Long, Y., Li, N. \& Ye, M. Soft Matter 8, 1831-1836 (2012).

29 Park, T. G. \& Hoffman, A. S. Sodium chloride-induced phase transition in nonionic poly (N-isopropylacrylamide) gel. Macromolecules 26, 5045-5048 (1993).

30 Burba, C. M., Carter, S. M., Meyer, K. J. \& Rice, C. V. Salt effects on poly(Nisopropylacrylamide) phase transition thermodynamics from NMR spectroscopy. J. Phys. Chem. B 112, 10399-10404 (2008).

31 Freitag, R. \& Garret-Flaudy, F. Salt effects on the thermoprecipitation of poly-(Nisopropylacrylamide) oligomers from aqueous solution. Langmuir 18 3434-3440 (2002)

(c) (1)(2) (2) This work is licensed under a Creative Commons Attribution-NonCommercial-ShareAlike 3.0 Unported License. The images or other third party material in this article are included in the article's Creative Commons license, unless indicated otherwise in the credit line; if the material is not included under the Creative Commons license, users will need to obtain permission from the license holder to reproduce the material. To view a copy of this license, visit http://creativecommons.org/licenses/by-nc-sa/3.0/

Supplementary Information accompanies the paper on the NPG Asia Materials website (http://www.nature.com/am) 